\title{
THE RADIAL VELOCITIES OF PLANETARY NEBULAE IN NGC 3379
}

\author{
ROBIN CIARDULLO \\ Department of Astronomy and Astrophysics, Penn State University, 525 Davey Lab, University \\ Park, Pennsylvania 16802, USA \\ and \\ GEORGE JACOBY \\ Kitt Peak National Observatory, National Optical Astronomy Observatories, P.O. Box 26732, \\ Tucson, Arizona 85726, USA
}

Several authors have analyzed the kinematics of elliptical galaxies using surface photometry in combination with absorption line velocity dispersion measurements. However, these analyses never explore the halos of galaxies, since the best absorption line measurements extend only $\sim 1 r_{e}$. The only way to extend our knowledge of stellar kinematics to larger radii is to use the emission lines of planetary nebula for radial velocity measurements.

In May 1991 we placed Kitt Peak's multi-fiber spectrograph (NESSIE) at the R-C focus of the Mayall $4 \mathrm{~m}$ telescope and measured the radial velocities of $29 \mathrm{PN}$ in the outer envelope of the normal elliptical galaxy NGC 3379. These observations achieved an internal precision of $\sim 17 \mathrm{~km} \mathrm{~s}^{-1}$, and allow us to measure the galaxy's mass out to a radius of $3 ! 5\left(3.5 r_{e}\right.$, or $\left.11 \mathrm{kpc}\right)$. Assuming that our PN trace the mass, then the projected mass estimator of Heisler, Tremaine, and Bahcall (1985) gives a value of $\sim 1.4 \times 10^{11} M_{\odot}$. The implied mass-to-light ratio, $M / L_{B} \sim 18$, is in agreement with that found from absorption line analyses and suggests that dark matter is not a major constituent of NGC 3379's halo.

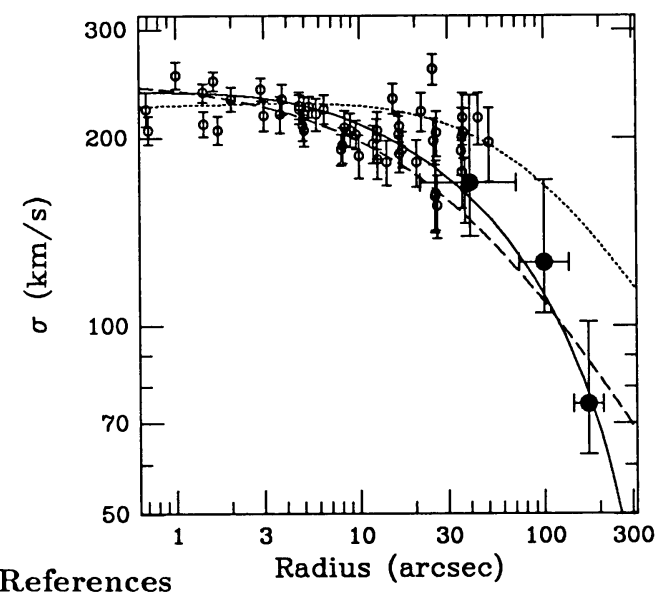

Figure 1. Displayed is the velocity dispersion profile for NGC 3379. The open circles are the absorption line measurements, mostly from Davies and Birkinshaw (1988); the filled circles show our PN data. For comparison, 3 constant mass-to-luminosity models are shown: an $r_{t} / r_{c} \sim 2.2$ King (1966) model (solid line), an isotropic orbit Jaffe (1983) model (dashed line), and a circular orbit Jaffe model (dotted line). A Jaffe model with radial orbits is ruled out by the data. Note that in general, isotropic orbits fit the data reasonably well.

Davies, R.L., and Birkinshaw, M. 1988, Ap. J. Suppl., 68, 409.

Heisler, J., Tremaine, S., and Bahcall, J.N. 1985, Ap. J., 298, 8. Jaffe, W. 1983, M.N.R.A.S., 202, 995.

King, I.R. 1966, A. J., 67, 471. 